\title{
Effect of smoking on retrobulbar blood flow in thyroid eye disease
}

A Sadeghi-Tari ${ }^{1}$, M Jamshidian-Tehrani ${ }^{1}$, A Nabavi ${ }^{1}$, S Sharif-Kashani ${ }^{2}$, E Elhami ${ }^{1}$, N Hassanpour ${ }^{1}$ and K Ameli Zamani ${ }^{1}$

\begin{abstract}
Purpose To evaluate the effect of smoking on retrobulbar blood flow parameters by color Doppler imaging in patients with thyroid eye disease.

Patients and methods In this observational case series, blood flow parameters in the ophthalmic artery, superior ophthalmic vein, central retinal artery, and vein were determined by color Doppler imaging in patients with thyroid eye disease. Patients were grouped as smokers and non-smokers. Never smokers and those who had stopped smoking for at least $\mathbf{1}$ year before onset of ophthalmopathy were considered as nonsmokers. A thorough ophthalmic examination including Hertel exophthalmometry was performed. Orbital CT scan was also carried out in all patients.

Results Fifty-one orbits from 30 patients between 21 and 62 years old (mean \pm SD: $40.8 \pm 12.0$ ) were enrolled in this study. Smokers had greater proptosis and more active and sever disease. $(P<0.05)$ Muscle involvement based on CT scan did not vary in smokers and non-smokers. Maximum velocity $(3.78 \pm 1.74$ vs $5.27 \pm 2.14, P<0.001$; $t$-test) and minimum velocity $(1.74 \pm 0.78 \mathrm{vs}$ $3.26 \pm 1.36, P=0.014$; $t$-test) in superior ophthalmic vein were significantly lower in smokers than non-smokers. Even after adjusting for age, sex, and clinical activity score and severity, smokers had a lower minimum velocity in superior ophthalmic vein ( $P=0.01$; ANCOVA analysis).

Conclusion Cigarette smoking may correlate with increase in orbital venous congestion in thyroid eye disease.
\end{abstract}

Eye (2016) 30, 1573-1578; doi:10.1038/eye.2016.184; published online 19 August 2016

\section{Introduction}

Thyroid eye disease (TED), also named as Graves' ophthalmopahty (GO), is an autoimmune disorder that shows ocular manifestations including eyelid retraction, proptosis, strabismus, optic neuropathy, conjunctival hyperemia, and chemosis. TED is most likely associated with Graves' disease but less frequently occurs in euthyroid and hypothyroid patients. ${ }^{1,2}$

Although fundamental pathogenesis of TED remained obscure, alteration in orbital connective tissue by autoimmune process has been studied more deeply. ${ }^{3,4}$ In addition, venous congestion may be responsible for some clinical features such as optic neuropathy and rise in intraocular pressure. ${ }^{5-7}$

Color Doppler imaging (CDI) is a noninvasive method which determines vascular flow characteristics, increasingly used in many orbital disorders including TED. Reduction in superior ophthalmic vein (SOV) flow rate is a well-known feature in TED, ${ }^{5-9}$ although some studies attributed this characteristic to especial types of TED. ${ }^{10}$ Alteration in other orbital vascular structures blood flow such as central retinal artery (CRA) and ophthalmic artery (OA) is less investigated and previous studies have resulted in conflicting data. ${ }^{11-13}$

Cigarette smoking is a well-documented risk factor that increases incidence, severity, and course duration of TED and associated with poor treatment response. Change in orbital soft tissues is one of the main proposed mechanisms in which smoking may affect the TED. ${ }^{14-16}$ However, role of smoking in increased venous congestion in TED was not examined yet. This study was conducted to evaluate retrobulbar blood flow parameters by CDI in patients with TED and to determine if smoking is correlated with the results.

\section{Material and methods}

This study is a prospective interventional case series conducted in Farabi Eye Hospital, Tehran between September 2013 and October 2014. Following the tenets of the Declaration of Helsinki, all participants provided informed constant. Local ethical board committee
${ }^{1}$ Eye Research Center, Farabi Eye Hospital, Tehran University of Medical Sciences, Farabi Hospital, Tehran, Iran

${ }^{2}$ Department of Radiology, Tehran University of Medical Sciences, Imam Khomeini Hospital, Tehran, Iran

Correspondence: M Jamshidian-Tehrani, Eye Research Center, Farabi Eye Hospital, Tehran University of Medical Sciences, Farabi Hospital, Qazvin Square, Tehran 1336616351, Iran Tel: +98 9171193010; Fax: +98 2155409095. E-mail: mansooreh_2468@ yahoo.com

Received: 12 January 2016 Accepted in revised form: 28 June 2016 Published online: 19 August 2016 
approval from the Tehran University of Medical Sciences was obtained.

The diagnosis of TED was made based on the presence of 2 out of 3 following criteria: clinical or laboratory signs of immune related thyroid dysfunction, typical orbital signs of the disease, and typical radiographic evidences of TED on CT scan. The diagnosis was confirmed in our ophthalmology department. All participants had normal thyroid function tests at the time of this study. Patients with history of other orbital disease, orbital surgery, or radiotherapy were excluded from the study.

A comprehensive ocular examination including best corrected distance visual acuity (BCVA), slit lamp biomicroscopy, Goldman tonometry, fundus evaluation, relative afferent pupilary response, extraocular motility, and Hertel exophthalmometry was performed in all participants. Visual field analysis with Swedish interactive threshold assay standard 24.2 programs also was done. Clinical activity score (CAS) was recorded based on EUGOGO guideline. ${ }^{17}$

According to CAS, patients were classified as active $(\mathrm{CAS} \geq 3)$ or inactive disease $(\mathrm{CAS}<3)$. Moderate to severe disease was defined by presence of at least one of the following signs or symptoms: exophthalmus $\geq 3 \mathrm{~mm}$ above normal, lid retraction $\geq 2 \mathrm{~mm}$ above normal, and moderate to severe soft tissue involvement. No patients had sign of dysthyroid optic neuropathy or corneal break down (which were considered as sight threatening) ${ }^{17}$

Cigarette smoking habits were recorded by a questionnaire. Participants were allocated into smokers and non-smokers groups. Patients who never smoked or had quitted smoking for at least 1 year before onset of eye involvement were considered as non-smoker. Current smokers or patients who stopped smoking for $<1$ year before onset of ophthalmopathy were considered as smokers. In addition, we documented the mean number of cigarettes consumption per day in current smokers.

CDI using $12 \mathrm{MHz}$ linear transducer (Voluson 730, General Electric, Milwaukee, WI, USA) was carried out in radiology department in Imam Khomeini hospital. CDIs were performed in supine position with tilted head in $30^{\circ}$. Ultrasound gel was applied on closed upper eyelids. Care was taken to minimize pressure on the globe. SOV was visualized at its distal portion before leaving orbit. Evaluation of OA blood flow was performed in about $2 \mathrm{~cm}$ behind the globe. CRA and central retinal vein (CRV) were identified within retrolaminar part of optic nerve. CDI parameters including maximum and minimum blood flow for SOV and CRV were measured. Arteries were analyzed by peak systolic velocity and end diastolic velocity. Average of at least three measurements was considered in each parameter. Resistance index (RI) was calculated as ((peak systolic velocity-end diastolic velocity)/peak systolic velocity).
Orbital CT scan in axial and coronal sections was also obtained. The largest diameter of each muscle in any midsection cuts was measured for further analysis. For the means of comparison with normative data, optic nerve diameter in cross sectional CT was regarded as normal for inferior and superior rectus measurements. Normal medial rectus is $\sim 1-1.5$ times thicker and lateral rectus is less thick than normal optic nerve. ${ }^{18} \mathrm{CDI}$ and orbital CT scan interpretation was done by the same blinded radiologist (S.S.K).

Variables are expressed as mean \pm SD or number (proportion). Independent Student's $t$-test or $X^{2}$ test was used to analyze variables between smoker and nonsmoker patients. Comparing blood flow parameters between patients with different amount of tobacco consumption was performed by one-way ANOVA analysis. To adjust confounding factors, analysis of covariance (ANCOVA)of the CDI parameters among smokers and non-smokers was done. Correlation between number of muscle involvement and CDI parameters was analyzed using Pearson's correlation coefficient.

Statistical analysis was performed using SPSS for Windows software version 16 (SPSS, Inc, Chicago, IL, USA). A $P$-value $<0.05$ was considered statistically significant.

\section{Results}

Fifty-one orbits from 30 patients with mean age of $40.8 \pm 12.0$ (range: $21-62$ ) years were enrolled in this study. Sixteen patients (31.4\%) were non-smoker and 35 patients $(68.6 \%)$ were considered as smoker.

Age, BCVA and IOP were statistically similar between smokers and non-smokers (Table 1). Smokers have greater

Table 1 Baseline characteristics in smokers and non-smokers

\begin{tabular}{lccc}
\hline Variables & $\begin{array}{c}\text { Smokers } \\
(\mathrm{n}=16)\end{array}$ & $\begin{array}{c}\text { Non-smokers } \\
(\mathrm{n}=35)\end{array}$ & P-value \\
\hline Age (years) & $41.2 \pm 10.7$ & $40.6 \pm 12.7$ & 0.86 \\
Sex (Male) & $10(62.5 \%)$ & $7(20.0 \%)$ & 0.003 \\
Laterality (OD) & $7(43.8 \%)$ & $19(54.3 \%)$ & 0.485 \\
$\begin{array}{l}\text { Thyroid function at } \\
\text { presentation (Graves' }\end{array}$ & $15(93.7 \%)$ & $31(88.6 \%)$ & 0.223 \\
disease) & & & \\
$\begin{array}{l}\text { BCVA (log MAR) } \\
\text { IOP (mm Hg) }\end{array}$ & $0.06 \pm 0.07$ & $0.09 \pm 0.011$ & 0.334 \\
Proptosis (mm) & $14.1 \pm 1.9$ & $14.2 \pm 1.6$ & 0.793 \\
CAS & $24.4 \pm 1.9$ & $22.3 \pm 2.8$ & 0.002 \\
$\quad<3$ & & & 0.01 \\
$\quad \leq 3$ & $9(43.8 \%)$ & $28(80.0 \%)$ & \\
$\quad$ Severity & & $7(20.0 \%)$ & \\
$\quad$ Mild & & & 0.007 \\
$\quad$ Moderate to severe & $11(68.8 \%)$ & $10(28.6 \%)$ & \\
\hline
\end{tabular}

Abbreviations: BCVA, best corrected visual acuity; IOP, Intraocular pressure. Values are mean $\pm \mathrm{SD}$ or number (proportion). $P$-values are for Student's $t$-test or $X^{2}$ between two groups. 
proportion of male patients than non-smokers. (62.5 vs $20 \%, P=0.001)$. Exophthalmometry measurements were $24.4 \pm 1.9$ in smokers and $22.3 \pm 2.8$ in non-smokers. $(P=0.002)$ Furthermore, smokers had more severe and active disease; proportion of patients with $C A S \geq 3$ was greater in smokers $(56.2 \%)$ than non-smokers (20\%). $\left(P=0.01, X^{2}\right.$ test). Moderate to severe disease was observed in $68 \%$ of smokers and $28.6 \%$ of non-smokers. The difference was statistically significant. $(P=0.007)$

Orbital CT scan was also performed for all except one non-smoker patients. Inferior rectus followed by medial rectus and superior rectus was the mostly involved muscles. However, proportion of lateral rectus enlargement is slightly higher than superior rectus in smokers. Neither involvement of specific rectus muscle nor number of rectus muscle enlargement differed between smokers and non-smokers patients. $(P>0.05$; Table 2).

All vessels found successfully except one SOV in smoker group. Orbital flow parameters in SOV, CRV, OA and CRA in smokers and non-smokers are presented in Table 3. Maximum velocity $(3.78 \pm 1.74$ vs $5.27 \pm 2.14$, $P=0.014)$ and minimum velocity $(1.74 \pm 0.78$ vs $3.26 \pm 1.36, P<0.001)$ in SOV were significantly lower in smokers than non-smokers. However, CDI parameters in other vessels were statistically similar in two groups.

After adjusting for age, sex, CAS and severity by

ANCOVA analysis, minimal velocity in SOV was still lower in smokers $(P=0.01)$.

Number of rectus muscle involvement in non-smoker group was inversely correlated with SOV minimum velocity $(r=-418, P=0.003)$. However, there is not any significant correlation between other CDI parameters and number of rectus muscle involvement in smoker or nonsmoker group $(P>0.05)$.

In current smokers (16 patients), six patients $(37.5 \%)$ smoked 1-10 cigarettes per day, six patients (37.5\%) smoked 11-20 cigarettes per day and four patients (25\%) smoked $<20$ cigarettes per day. Maximal velocity in SOV tends to decrease with higher cigarettes smoking. However, the difference was not statistically significant $(P=0.054)$. Other CDI parameters did not significantly vary in patients with different amount of current tobacco consumption (Table 4).

\section{Discussion}

To our knowledge, role of smoking in alteration of orbital blood flow in patients with TED was not examined exclusively. Our results revealed that smoking may play an independent role in venous congestion in patients with TED. Maximal and minimal venous pressure of SOV was significantly lower in smokers. Even after adjusting for other confounding factors, including severity and activity,
Table 2 Rectus muscles involvement based on CT scan in smokers and non-smokers

\begin{tabular}{lccc}
\hline Variables & $\begin{array}{c}\text { Smokers } \\
(\mathrm{n}=16)(\%)\end{array}$ & $\begin{array}{c}\text { Non-smokers } \\
(\mathrm{n}=34)(\%)\end{array}$ & P-value \\
\hline $\begin{array}{l}\text { Rectus muscle involvement } \\
\quad\end{array}$ & $8(50)$ & $18(52.9)$ & 0.924 \\
$\quad$ SR involvement & $13(81.2)$ & $29(85.3)$ & 0.711 \\
IR involvement & $13(81.2)$ & $22(64.7)$ & 0.182 \\
MR involvement & $9(56.2)$ & $15(44.1)$ & 0.423 \\
LR involvement & & & \\
& & & \\
Number of muscle involvement & 0 & $2(5.9)$ & 0.331 \\
No muscle involvement & $1(6.2)$ & $7(20.6)$ & \\
One muscle involvement & $8(50.0)$ & $11(32.4)$ & \\
Two muscle involvement & $6(37.5)$ & $12(35.3)$ & \\
Three muscle involvement & $6(5.9)$ & $2(5.9)$ & \\
Four muscle involvement & $1(6.2)$ & \\
\hline
\end{tabular}

Abbreviations: IR, inferior rectus; LR, Lateral rectus; MR, medial rectus; SR, superior rectus. $P$-values are for $X^{2}$ or Fisher's exact test between two groups.

Table 3 CDI parameters in smokers and non-smokers

\begin{tabular}{llcr}
\hline Variables & $\begin{array}{c}\text { Smokers } \\
(\mathrm{n}=16)\end{array}$ & $\begin{array}{c}\text { Non-smokers } \\
(\mathrm{n}=35)\end{array}$ & P-value \\
\hline OA & & & \\
$\quad$ Peak systolic velocity (cm/s) & $28.9 \pm 6.12$ & $30.72 \pm 7.11$ & 0.381 \\
End diastolic velocity $(\mathrm{cm} / \mathrm{s})$ & $7.09 \pm 2.6$ & $7.74 \pm 2.38$ & 0.379 \\
RI & $0.75 \pm 0.07$ & $0.74 \pm 0.05$ & 0.705 \\
& & & \\
CRA & & & \\
Peak systolic velocity (cm/s) & $8.91 \pm 2.83$ & $10.21 \pm 3.48$ & 0.199 \\
End diastolic velocity (cm/s) & $2.54 \pm 1.33$ & $3.23 \pm 1.8$ & 0.181 \\
RI & $0.67 \pm 0.16$ & $0.68 \pm 0.15$ & 0.607 \\
& & & \\
SOV & & & \\
Maximal velocity (cm/s) & $3.78 \pm 1.74$ & $5.27 \pm 2.14$ & 0.014 \\
Minimal velocity (cm/s) & $1.74 \pm 0.78$ & $3.26 \pm 1.36$ & $<0.001$ \\
& & & \\
CRV & & & \\
Maximal velocity $(\mathrm{cm} / \mathrm{s})$ & $4.44 \pm 1.02$ & $4.6 \pm 0.75$ & 0.549 \\
Minimal velocity $(\mathrm{cm} / \mathrm{s})$ & $2.74 \pm 0.62$ & $2.96 \pm 0.78$ & 0.327 \\
\hline
\end{tabular}

Values are shown as mean $\pm \mathrm{SD}$. $P$-values are for Student's $t$-test between two groups.

smoking is associated with lower SOV minimal venous pressure. Furthermore, there was a non-significant trend toward decrease in SOV maximal velocity with higher amount of cigarette smoking per day.

Venous congestion plays a key role in pathogenesis of TED. Conjunctival hyperemia and chemosis, increased in episcleral venous pressure and optic nerve compression are related, in part, to decrease venous outflow. ${ }^{5-7}$ Pervious investigations showed a decrease in blood flow of SOV in TED. ${ }^{8,9,19}$ Alp et al ${ }^{8}$ studied 111 patients with Graves' disease and 46 normal subjects. Authors found 
Table 4 CDI parameters in smokers based on current cigarette smoking per day

\begin{tabular}{|c|c|c|c|c|}
\hline Variables & 1-10 cigarettes/day $(\mathrm{n}=6)$ & $11-20$ cigarettes/day $(n=6)$ & $20<$ cigarettes/day $(\mathrm{n}=4)$ & P-value \\
\hline \multicolumn{5}{|l|}{$O A$} \\
\hline Peak systolic velocity $(\mathrm{cm} / \mathrm{s})$ & $27.14 \pm 1.81$ & $30.98 \pm 5.07$ & $28.41 \pm 11.14$ & 0.578 \\
\hline End diastolic velocity $(\mathrm{cm} / \mathrm{s})$ & $5.82 \pm 1.98$ & $8.37 \pm 2.65$ & $7.06 \pm 3.01$ & 0.249 \\
\hline RI & $0.79 \pm 0.07$ & $0.73 \pm 0.07$ & $0.74 \pm 0.06$ & 0.432 \\
\hline \multicolumn{5}{|l|}{$C R A$} \\
\hline Peak systolic velocity (cm/s) & $8.41 \pm 3.12$ & $9.5 \pm 3.04$ & $8.79 \pm 2.7$ & 0.819 \\
\hline End diastolic velocity $(\mathrm{cm} / \mathrm{s})$ & $2.32 \pm 1.11$ & $2.3 \pm 1.4$ & $3.23 \pm 1.64$ & 0.520 \\
\hline RI & $0.7 \pm 0.16$ & $0.76 \pm 0.12$ & $0.6 \pm 0.21$ & 0.317 \\
\hline \multicolumn{5}{|l|}{ SOV } \\
\hline Maximal velocity (cm/s) & $4.64 \pm 1.31$ & $3.69 \pm 1.21$ & $2.61 \pm 1.12$ & 0.054 \\
\hline Minimal velocity (cm/s) & $1.9 \pm 0.75$ & $1.79 \pm 0.88$ & $1.43 \pm 0.79$ & 0.67 \\
\hline \multicolumn{5}{|l|}{$C R V$} \\
\hline Maximal velocity (cm/s) & $3.86 \pm 0.73$ & $5.01 \pm 1.29$ & $4.49 \pm 0.54$ & 0.159 \\
\hline Minimal velocity $(\mathrm{cm} / \mathrm{s})$ & $2.58 \pm 0.51$ & $2.97 \pm 1.01$ & $2.61 \pm 0.47$ & 0.623 \\
\hline
\end{tabular}

Values are shown as mean \pm SD. P-values are for one-way ANOVA analysis between three groups.

that maximal and minimal velocity in SOV in patients with Graves' ophthalmopathy was significantly lower than patients with Graves' disease without ophthalmopathy and normal controls. Correlation between reduced venous outflow and disease activity was also noted in some studies. ${ }^{8,12}$ Yanik et al ${ }^{12}$ found a significantly difference between maximal SOV velocity in patients with different CAS values. They found that patients with CAS value 3 or above had a lower maximal velocity in SOV than patients with lower CAS values. External compression may be the main cause of venous obstruction in TED. Other factors such as elevated systemic blood pressure or intraocular pressure, which may be present in Graves' disease, might not affect venous outflow status in TED. ${ }^{8,20}$ Somer et al ${ }^{9}$ showed that decrease in SOV blood flow velocity is linked to apical crowding and the coexistence of horizontal and vertical extraocular muscle involvement in CT scan. However, Alp et al ${ }^{8}$ did not observe a correlation between muscle index and SOV blood flow, and proposed that other factor may contributed to increase congestion in TED.

Tobacco smoking has a variety of effects in thyroid function; however, role of smoking in Graves' disease and TED is more pronounced. Orbital involvement may occur in as high as threefolds greater in smokers and they may have more severe and prolonged ophthalmopathy. Smoking is linked to exacerbation of ophthalmopathy after 131I therapy. Moreover, smoking has a negative correlation with outcome of treatment in TED. ${ }^{14,16}$ In present study, smokers had greater amount of proptosis and more active and sever disease than non- or exsmokers, consistent with previous literature. ${ }^{21}$
Although association between smoking and TED is well established, underlying mechanisms were poorly understood. Smoking may induce hypoxia and elaborate inflammatory cytokines in orbit. ${ }^{14,15}$ One study showed that cigarette smoke extract in association with inflammatory cytokines have a synergic effect in adipogenesis of orbital fibroblasts. ${ }^{22}$ Szucs-Farkas et al ${ }^{23}$ found that soft tissue volumes were correlated with smoking history, whereas extraocular muscle volumes were not related. In contrast, Regensburg et al ${ }^{24}$ studied 95 patients with untreated GO and found that smoking is associated with an increase in extraocular muscle volume but not fat volume. Herein, rectus muscle involvement based on qualitative CT scan analysis was similar in smokers and non-smokers. In addition, number of rectus muscle involvement was inversely correlated with SOV minimum blood flow only in non-smoker group. Contribution of extraocular muscle involvement and decrease in SOV blood flow is mentioned in some other studies. ${ }^{9,25}$ However, we did not evaluate the exact orbital muscle or fat volume. So, reduced SOV blood flow in smokers in our study may still relate, in part, to alteration in orbital muscle or fat volume. Regarding the independence of SOV minimum velocity in smokers and disease severity, possible other mechanisms might participate.

We did not find a statistically difference in CDI parameters of OA, CRA and CRV between smokers and non-smokers. Some studies illustrated that enhanced orbital inflammation in TED may result in elevated arterial blood flow which correlated with disease activity. ${ }^{12,19}$ Conversely, Perez-Lopez et al ${ }^{13}$ reported that RIs in the CRA and OA were significantly higher in 
patients with GO than in the controls. Finally, consistent to our findings, Benning et $a l^{26}$ did not observed any significant difference in blood flow velocity in CRA between patients with GO and the control group.

We grouped patients who quitted smoking 1 year before onset of orbital involvement as non-smoker. Regensburg et al $^{24}$ also had similar grouping in examining association between smoking and muscle volume in Graves' ophthalmopathy. This method was based on studies indicating a reversibility of smoking effect in TED. Pfeilschifter and Ziegler ${ }^{27}$ followed patients for at least 1 year after onset of Graves' disease. They reported that exsmokers had a lower risk of developing ophthalmic involvement than current smokers even in comparable lifetime tobacco consumption.

In conclusion, this study illustrates a new aspect of smoking consequence in patients with TED. Cigarette smoking may correlate with increase in orbital venous congestion. One of the main limitations of our study was a relatively small number of smokers which prevents further subgroup analysis to assess more powerful doserelated effect. Furthermore, we did not evaluate extraocular muscle volume in quantitative detail, which might be an additional confounder of smoking effects in orbital blood flow. Further investigation is needed to assess the exact mechanism of smoking associated TED.

\section{Summary}

\section{What was known before}

- Cigarette smoking is a well-documented risk factor that increases incidence, severity, and course duration of TED. Venous congestion plays a key role in pathogenesis of TED.

\section{What this study adds}

- Cigarette smoking may correlate with increase in orbital venous congestion.

\section{Conflict of interest}

The authors declare no conflict of interest.

\section{Acknowledgements}

This study was supported financially by the vice chancellery of research of Tehran University of Medical Sciences.

\section{References}

1 Bartley GB, Fatourechi V, Kadrmas EF, Jacobsen SJ, Ilstrup DM, Garrity JA et al. Clinical features of Graves' ophthalmopathy in an incidence cohort. Am J Ophthalmol 1996; 121(3): 284-290.

2 McAlinden C. An overview of thyroid eye disease. Eye Vision 2014; 1: 9.

3 Shan SJ, Douglas RS. The pathophysiology of thyroid eye disease. J Neuro-ophthalmol 2014; 34(2): 177-185.

4 Weetman AP. Thyroid-associated eye disease: pathophysiology. Lancet 1991; 338(8758): 25-28.

5 Konuk O, Onaran Z, Ozhan Oktar S, Yucel C, Unal M. Intraocular pressure and superior ophthalmic vein blood flow velocity in Graves' orbitopathy: relation with the clinical features. Graefe's Arch Clin Exp Ophthalmol 2009; 247(11): 1555-1559.

6 Nakase Y, Osanai T, Yoshikawa K, Inoue Y. Color Doppler imaging of orbital venous flow in dysthyroid optic neuropathy. Jpn J Ophthalmol 1994; 38(1): 80-86.

7 Onaran Z, Konuk O, Oktar SO, Yucel C, Unal M. Intraocular pressure lowering effect of orbital decompression is related to increased venous outflow in Graves orbitopathy. Curr Eye Res 2014; 39(7): 666-672.

8 Alp MN, Ozgen A, Can I, Cakar P, Gunalp I. Colour Doppler imaging of the orbital vasculature in Graves' disease with computed tomographic correlation. Br J Ophthalmol 2000; 84(9): 1027-1030.

9 Somer D, Ozkan SB, Ozdemir H, Atilla S, Soylev MF, Duman S. Colour Doppler imaging of superior ophthalmic vein in thyroid-associated eye disease. Jpn J Ophthalmol 2002; 46(3): 341-345.

10 Monteiro ML, Angotti-Neto H, Benabou JE, Betinjane AJ. Color Doppler imaging of the superior ophthalmic vein in different clinical forms of Graves' orbitopathy. Jpn J Ophthalmol 2008; 52(6): 483-488.

$11 \mathrm{Li} \mathrm{H}$, Liu YH, Li DH, Zhang Y. [Value of measurements of blood flow velocity in central retinal artery in thyroidassociated ophthalmopathy]. Zhongguo yi xue ke xue yuan xue bao Acta Academiae Medicinae Sinicae 2004; 26(4): 460-462.

12 Yanik B, Conkbayir I, Acaroglu G, Hekimoglu B. Graves' ophthalmopathy: comparison of the Doppler sonography parameters with the clinical activity score. J Clin Ultrasound 2005; 33(8): 375-380.

13 Perez-Lopez M, Sales-Sanz M, Rebolleda G, Casas-Llera P, Gonzalez-Gordaliza C, Jarrin E et al. Retrobulbar ocular blood flow changes after orbital decompression in Graves' ophthalmopathy measured by color Doppler imaging. Invest Ophthalmol Vis Sci 2011; 52(8): 5612-5617.

14 Wiersinga WM. Smoking and thyroid. Clin Endocrinol (Oxf) 2013; 79(2): 145-151.

15 Chng CL, Lai OF, Chew CS, Peh YP, Fook-Chong SM, Seah LL et al. Hypoxia increases adipogenesis and affects adipocytokine production in orbital fibroblasts-a possible explanation of the link between smoking and Graves' ophthalmopathy. Int J Ophthalmol 2014; 7(3): 403-407.

16 Hegedius L, Brix TH, Vestergaard P. Relationship between cigarette smoking and Graves' ophthalmopathy. J Endocrinol Invest 2004; 27(3): 265-271.

17 Bartalena L, Baldeschi L, Dickinson A, Eckstein A, Kendall-Taylor P, Marcocci C et al. Consensus statement of the European Group on Graves' orbitopathy (EUGOGO) on management of GO. Eur J Endocrinol 2008; 158(3): 273-285.

18 K-Jafari A, Sadeghi-Tari A, Minaee-Noshahr N, Ameri A, Anvari F, Ali-Mahmoudi A et al. Ocular movement disorders and extraocular muscle involvement in Iranian Graves' 
ophthalmopathy patients. Binocul Vis Strabismus Q 2010; 25(4): 217-230.

19 Monteiro ML, Moritz RB, Angotti Neto H, Benabou JE. Color Doppler imaging of the superior ophthalmic vein in patients with Graves' orbitopathy before and after treatment of congestive disease. Clinics 2011; 66(8): 1329-1334.

20 Kurioka Y, Inaba M, Kawagishi T, Emoto M, Kumeda Y, Inoue $\mathrm{Y}$ et al. Increased retinal blood flow in patients with Graves' disease: influence of thyroid function and ophthalmopathy. Eur J Endocrinol 2001; 144(2): 99-107.

21 Vestergaard P. Smoking and thyroid disorders-a metaanalysis. Eur J Endocrinol 2002; 146(2): 153-161.

22 Cawood TJ, Moriarty P, O'Farrelly C, O'Shea D. Smoking and thyroid-associated ophthalmopathy: A novel explanation of the biological link. J Clin Endocrinol Metab 2007; 92(1): 59-64.

23 Szucs-Farkas Z, Toth J, Kollar J, Galuska L, Burman KD, Boda $\mathrm{J}$ et al. Volume changes in intra- and extraorbital compartments in patients with Graves' ophthalmopathy: effect of smoking. Thyroid 2005; 15(2): 146-151.

24 Regensburg NI, Wiersinga WM, Berendschot TT, Saeed P, Mourits MP. Effect of smoking on orbital fat and muscle volume in Graves' orbitopathy. Thyroid 2011; 21(2): 177-181.

25 da Rocha Lima B, Perry JD. Superior ophthalmic vein enlargement and increased muscle index in dysthyroid optic neuropathy. Ophthal Plast Reconstr Surg 2013; 29(3): 147-149.

26 Benning H, Lieb W, Kahaly G, Grehn F. [Color duplex ultrasound findings in patients with endocrine orbitopathy]. Der Ophthalmologe 1994; 91(1): 20-25.

27 Pfeilschifter J, Ziegler R. Smoking and endocrine ophthalmopathy: impact of smoking severity and current vs lifetime cigarette consumption. Clin Endocrinol 1996; 45(4): $477-481$. 